\title{
Adgrf5 contributes to patterning of the endothelial deep layer in retina
}

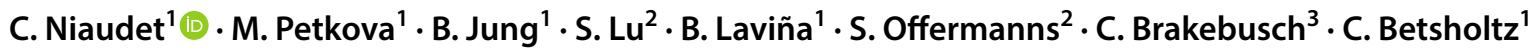

Received: 10 January 2019 / Accepted: 17 June 2019 / Published online: 29 June 2019

(c) The Author(s) 2019

\begin{abstract}
Neovascularization of the inner retinal space is a major cause of vision loss. In retinal angiomatous proliferation (RAP) syndrome, newly formed vessels originate from the retinal plexus and invade the inner retinal space. However, the molecular pathways preventing subretinal vascularization remain largely unknown. In most murine models of RAP, pathological neovascularization occurs concomitantly with the development of the retinal vasculature. Here, we demonstrate that disturbing the sequence of morphogenetic events that shape the three-layered retinal vascular network leads to subretinal vascularization. Sprouts emerging from the perivenous region after the first postnatal week extended toward the retinal space where they merged into the deep layer. The small GTPase Rac1 was required for the formation of these vascular extensions and the vascular inner plexus is formed coaxially to the overarching veins. The adhesion receptor Adgrf5 was highly expressed in the endothelium of the central nervous system, where it regulates blood-brain barrier formation. The vascular superficial plexus of Adgrf5 mutant mouse retinae exhibited an increased vascular density in the perivenous areas with increased projections toward the inner plexus where they subsequently created hyper-dense endothelial cells (EC) clusters. Disturbing the perivenous pool of EC thus significantly altered the inner plexus formation. These abnormalities culminated in transient vascular protrusions in the inner retinal space. Taken together, these results reveal a previously unobserved vascular morphogenetic defect in Adgrf5 knockout mice, implicating a role for ADGRF5 in the initiation of subretinal vascularization. Our findings also illustrate how vein-derived EC shape the inner retinal layer formation and could control the appearance of angiomatous malformations.
\end{abstract}

Keywords Retina $\cdot$ Adhesion receptor $\cdot$ Adgrf5

\section{Introduction}

The visual function of the eye is dependent on a strict organization of retinal tissue. The retina exhibits a multi-layered structure, in which the different cellular compartments and the vascular beds are organized to allow tissue oxygenation with minimal blockade of the passage of light. Thus, the neural retinal layer is ensheathed between two separate vascular beds. On the posterior side, the plexus of choriocapillaries is the main oxygen supplier of the outer layers of the retina. On the inner (vitreal) side, the retinal vascular network develops into a stereotypical tri-layered architecture. The surface vascular plexus extends over the ganglionic cell layer (GCL) while two intra-retinal capillary networks radiate above the inner plexiform layer (IPL) and the outer plexiform layer (OPL), respectively [1,2].

The retinal vasculature first develops as a primary plexus sprouting from the optic nerve across the surface of the 
GCL. In the mouse, this process commences after birth. The superficial plexus expands and fully covers the retina by postnatal day 7 (P7). Numerous and detailed mechanisms that regulate this initial phase, including vascular endothelial growth factor (VEGF) and Notch signaling, have been well studied [3]. Soon thereafter, new sprouts expand into the retinal space to successively form the inner and the intermediary plexus, at $\mathrm{P} 7$ and $\mathrm{P} 12$, respectively (summarized in Fig. 1, "Physiological") [2]. Major molecular cues guiding this second step have been proposed [4-6]. For example, VEGF secreted by the photoreceptor cells is the major driver of the deep layer formation. A progressive decrease in VEGF receptor 2 (VEGFR2) expressed by neurons sets the pace for blood vessel invasion, while local cues expressed by neighboring cell types constrain the expansion of the intermediary and the deep plexus [7].

Despite the extensive vasculature in the area, the photoreceptor layer is entirely devoid of any blood vessel under physiological circumstances. Currently, it remains unknown whether the avascular privilege in the photoreceptor layer has any biological function. A recent study has suggested that a hypoxic niche might be critical for proper neuroretina development by maintaining a pool of stem cells [7]. Moreover, vascular invasion of the neuroretinal space hampers optimal photo-transduction and can lead to irreversible blindness. Pathological invasion of the avascular inner retinal space may occur from either the choroidal or the retinal vascular network, leading to the conditions known as agerelated macular degeneration (AMD) and retinal angiomatous proliferation (RAP), respectively. Both conditions cause severe impairment of the functionality of the retina and will ultimately damage vision $[8,9]$. In patients affected by RAP, vascular sprouts first emerge from the deep vascular plexus above the OPL, bud inside the inner retinal space, and finally anastomose to the choroidal vasculature in the final stage of the pathogenic process (see Fig. 1, "Pathological") [10, 11].

Unlike AMD, very few mutations have been linked with RAP in patients. Several loss-of-function mutant mice exhibit subretinal neovascularization, which evolves in the most severe cases into RAP. These studies suggest that the mechanisms leading to subretinal neovascularization might be closely associated with the ones governing normal deep retinal plexus development. Indeed, subretinal neovascularization often occurs in a narrow time window in mice, around P14 [12-16]. This coincides with a residual plasticity of retinal vascular development, where the vessels from the superficial plexus layer begin to project down to generate the nascent deeper plexus layer. The descending vessels are funneled through the action of local cues such as semaphorins and disturbing these cues results in hypovascularization [4-6]. Conversely, altering the distribution of VEGF leads to subretinal neovascularization, which evolves into a full RAP syndrome. Physiologically, a rigorous protection against VEGF is maintained by the steady production of sFlt1 from the neuroretina [17].

Control of cellular polarity is essential to retinal morphogenesis [18]. Adhesion G protein-coupled receptors (aGPCRs) have been shown to control different types of polarity-related functions [19] such as oriented cell division [20], planar cell polarity [21, 22], and cell migration [23]. As such, aGPCRs are involved in regulating numerous developmental processes [24]. aGPCRs exert a part of their biological action through a long extracellular $N$-terminus bearing adhesive domains. These domains allow the receptors to interact with membrane-tethered partners or molecules embedded within the extracellular matrix, a feature that renders them unique within the GPCR superfamily [25, 26]. Adgrf5 is one of the 5 aGPCRs highly expressed in the central nervous system (CNS) endothelium [27]. Suppression of Adgrf5 in the endothelium impairs the function of the blood-brain barrier (BBB), but no morphogenetic defect in the CNS capillaries has been documented thus far [28]. In parallel, Adgrf5 knockout (KO) mice exhibit overproduction of surfactant in the lungs, linked with the expression of Adgrf5 in the type II alveolar epithelial cells [29-31].

In the present study, we assessed the impact of Adgrf5 deficiency on the formation of the inner vascular layer in the retina. Our results demonstrate that subretinal

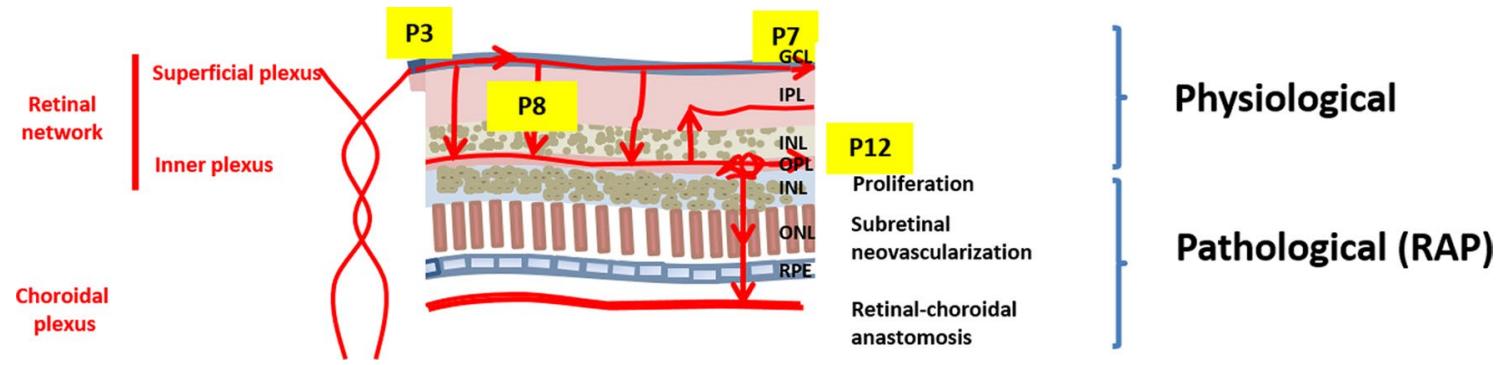

Fig. 1 Retinal vascular network development. Scheme summarizing the development of the retinal network ( $G C L$ ganglional cell layer, $I P L$ inner plexiform layer, $I N L$ inner nuclear layer, $O P L$ outer plexi- form layer, $O N L$ outer nuclear layer, $R P E$ retinal pigment epithelium). The lower scheme shows the progressive development of RAP lesions 
vascularization is intimately associated with the sequence of morphogenetic events that shape the superficial plexus. The vascular inner plexus developed coaxially to the overarching veins. In Adgrf5 $\mathrm{KO}$ mice, the vascular superficial plexus exhibits increased vascular density in the perivenous areas, creating increased EC projections toward the inner plexus where they form hyper-dense cell clusters. These abnormalities culminate in the formation of transient vascular protrusions in the inner retinal space. Taken together, these results illustrate how vein-derived EC contribute to the inner retinal layer formation and control the emergence of angiomatous proliferation. Our data also show the first vascular morphogenetic defect observed in Adgrf5 KO mice, and suggest that ADGRF5 deficiency mimics the initiation step of RAP.

\section{Materials and methods}

\section{Ethics statement}

Animal housing, as well as the experiments performed, was in accordance with Swedish legislation and was approved by the local animal ethics committees prior to experimentation. The protocols included in this study were approved by the Uppsala Committee (permit number C224/12). Animal suffering was minimized, and all surgical procedures were performed under anesthesia (Ketamine $(75 \mathrm{mg} / \mathrm{kg}$ ) and Dexdomitor $(0.5 \mathrm{mg} / \mathrm{kg}))$.

\section{Mouse models}

\section{Adgrf5 knockout}

Adgrf5 KO mice have been described previously [28]. Briefly, the Gpr116 ${ }^{-1-}$ Velocigene mouse line was generated by Regeneron using a VelociGene approach [32] to delete exon 3 to exon 21. Heterozygous mice were backcrossed to C57BL6 mice, for at least six generations. Knockout and heterozygous pups were identified through genotyping PCR using the following primers: forward: 5'-GGGTAACGTGCT CTCTCTGC-3', reverse wildtype: 5'-TGAACTCCTGGA TACTAGCC-3', or reverse knockout: 5'-TCATTCTCAGTA TTGTTTTGCC-3'). The predicted amplicon sizes are 325 base pairs (bp) for the Adgrf5 wild-type (WT) band, and $401 \mathrm{bp}$ for the knockout allele.

\section{Claudin5-GFP}

The transgenic mouse $C l d n 5$-GFP, expressing eGFP under the control of the Claudin5 promoter, was generated using Bacterial artificial chromosome (BAC) technology. Complete characterization of this endothelial transgenic line will be presented in an independent manuscript. The line was kept in a C57/B16 background ( $C l d n 5-\mathrm{GFP})$ or bred with the Adgrf5 KO (Adgrf5 KO $\times$ Cldn5-GFP).

\section{Rac1 ECKO}

Endothelium-specific tamoxifen-inducible Rac1 knockout mice (referred to as "Racl ECKO") were obtained by crossing $R a c I^{\text {flox/flox }}$ mice (floxed exon 3) [33] with the endothelial-specific $C d h 5$ (PAC)-CreERT2 mice [34]. To evaluate the recombination efficiency, Racl ECKO mice were bred into the Rosa26-EYFP reporter mouse [35]. Postnatal gene deletion was induced through maternal milk by tamoxifen (Sigma-Aldrich, \#T5648) gavage of the dam from P1 to P3. Mice were sacrificed at P8.

Racl ECKO pups were identified through genotyping using the following primers: forward 5'-TCACTGCTTGTC TCATGC A-3', reverse 5'-AACGTATGGTTTCATTAA ACTGA- $3^{\prime}$. The predicted amplicon size is $318 \mathrm{bp}$ for the floxed Racl allele and 236 bp for the WT allele. Cre-positive pups were identified through the following primers: forward: 5'-GATATC TCACGTACTGACGG-3', reverse 5'-TGACCA GAGTCATCCTTAGC-3'. The predicted amplicon size is $300 \mathrm{bp}$ for the Cre-positive band.

\section{Adgrf5 ECKO}

As described previously, Adgrf5 floxed exon 17 mice, generated by Novartis [29], were crossed to mice expressing a constitutive Tie2-Cre allele [36]. Offspring was intercrossed to generate mice homozygous for the floxed Gpr116 allele, and carrying one copy of Tie2-Cre. The male offspring was crossed to ROSA26mT/mG females (Jackson Laboratory, Stock No. 007576) to allow tracking of the recombined endothelial cells. This line, Flox Gpr116×Tie2Cre $\times$ ROSA26mT/mG, is referred to as "Gprl16 ECKO."

\section{Adrgf5 $\Delta 17$}

Adgrf5 $\Delta 17$ mice, harboring a deletion into the Adgrf5 exon 17 , which encodes for the transmembrane domains, were generated by germ line deletion. Briefly, a male carrying the floxed exon 17 was crossed to a Tie2-Cre-positive female mouse, since the activation of Cre recombinase by the Tie 2 promoter occurs during oocytogenesis. Thus, the floxed exon will be removed in the germ line, ultimately resulting in global deletion of the gene [37]. The efficiency of the LoxP/ Cre recombination in the offspring was examined by PCR to select mutants that have lost exon 17 using the following primers: forward 5'- TGTTAACCTCTGGCCTCTGC-3', reverse 5'- TGTTAACCTCTGGCCTCTGC-3' [29]. The predicted amplicon size is $490 \mathrm{bp}$. 


\section{Gpr116-mCherry}

Transgenic mice expressing mCherry under the control of the Gprl16 promoter (Gprl16-mCherry) were generated as described before using the BAC clone RP24-510M8 (CHORI, CA, USA) from mouse chromosome 17 containing the Gprl16 gene [38]. For genotyping by PCR the following primers were used: forward: 5'-CTTCATCATGTCCAC AGAACC-3'; reverse: 5'-AGGATGTCCCAGGCGAAG G-3'; PCR products of $502 \mathrm{bp}$ indicate the transgenic allele.

\section{Adgrf5 ECGOF}

The gain-of-function $\mathrm{Gt}($ Rosa26-CAG-Adgrf5) mouse model expresses a conditional knock-in allele of Adgrf5 under the control of a CAG promoter (Taconic).

To overexpress Adgrf5 in the endothelium, a Gt(Rosa26$C A G$-Adgrf5) mouse was crossed to the Tie2-Cre line to yield "Adgrf5 endothelial cell gain-of-function" (Adgrf5 ECGOF) mice. The transgene was detected using the following primers: forward 5'-TGGCAGGCTTGAGATCTGG-3', reverse 5'-CCCAAGGCACACAAAAAACC-3', control forward primer 5'-GGGGCAATCAATTGAGGG-3', control reverse primer 5'-CAACCTCTGCTTGGTTCTGG-3'. PCR products of $492 \mathrm{bp}$ indicate the presence of the conditional knock-in allele and the $333 \mathrm{bp}$ control band the presence of a WT allele.

\section{Tracer injection}

To assess cerebral vascular leakage, $1 \mathrm{kDa}$ Alexa Fluor 555-conjugated cadaverine (Life Technologies) was injected intravenously into the tail vein in adult mice ( 2 months). After 2 hours (h), the anesthetized animals were perfused with Hanks' balanced salt solution (HBSS) for 5 minutes (min), brains were harvested and homogenized in $1 \%$ Triton $\mathrm{X}-100$ in PBS (pH 7.2). Brain lysates were centrifuged at $13,000 \mathrm{rpm}$ for $20 \mathrm{~min}$ at $4{ }^{\circ} \mathrm{C}$ and the relative fluorescence of the supernatant was measured on a Synergy HT271167 plate reader (excitation/emission 540/590 nm). After HBSS perfusion, tracer extravasation into brain parenchyma was visualized with a Leica stereomicroscope [39].

\section{Organ processing}

Mice were anesthetized, and intracardiac perfusion of HBSS was performed.

For retinal whole mount staining, retinae were fixed in 4\% PFA in PBS for $1 \mathrm{~h}$ at room temperature (RT), dissected, permeabilized at $4{ }^{\circ} \mathrm{C}$ overnight, rinsed in PBS, washed twice in PBlec (1\% Triton-X100, $0.1 \mathrm{mM} \mathrm{CaCl}_{2}$, $0.1 \mathrm{mM} \mathrm{MgCl}_{2}, 0.1 \mathrm{mM} \mathrm{MnCl}{ }_{2}$ in PBS, pH 6.8), and incubated for $48 \mathrm{~h}$ at $4{ }^{\circ} \mathrm{C}$ with Alexa Fluor ${ }^{\circledR}$ 647-conjugated isolectin B4 (Invitrogen), as well as primary antibodies (CD31(Abcam), Erg (Abcam), ASMA (Sigma-Aldrich), all 1:500). After three washes in PBS, retinae were incubated for $2 \mathrm{~h}$ at RT with secondary antibodies, 1:400 diluted in PBS, $0.5 \%$ bovine serum albumin (BSA), and $0.25 \%$ Triton $\mathrm{X}-100$. Retinas were washed and flat-mounted in ProLong Gold mounting medium (Life Technologies).

For retinal histology, eyeballs were fixed in $4 \%$ PFA in PBS for $24 \mathrm{~h}$ at $4{ }^{\circ} \mathrm{C}$. They were subsequently embedded in paraffin and $5 \mu \mathrm{m}$ sections were prepared using a HM355S microtome (Thermo Fisher Scientific). After deparaffinization, sections were stained with eosine and with Mayer's hematoxylin. Finally, slides were dehydrated and mounted in Neo-Mount (Merck). Alternatively, dehydrated slides were post-fixed in $4 \%$ PFA for $1 \mathrm{~h}$, permeabilized for $2 \mathrm{~h}$, and incubated overnight with primary antibodies (collagen IV (AbD Serotec Bio-Rad), NeuN (Merck), GFAP (Zymed labs), TER-119 (BioLegend), all 1:100), and Alexa Fluor ${ }^{\circledR}$ 647-conjugated isolectin B4. After three washes in PBS, the sections were incubated for $2 \mathrm{~h}$ at RT with secondary antibodies and Hoechst, and mounted in ProLong Gold mounting medium (Life Technologies).

For lung histology, the trachea was exposed and a 20 -gauge blunt needle was inserted, perfused with a $4 \%$ PFA solution to inflate the lungs, which were post-fixed overnight in 4\% PFA solution. For paraffin sections, lungs were embedded in paraffin and 8 - $\mu$ m-thick sections were prepared using a HM355S microtome. After deparaffinization, sections were incubated $10 \mathrm{~min}$ in Mayer's hematoxylin, followed by eosin. Finally, slides were dehydrated and mounted in Neo-Mount (Merck).

\section{Confocal microscopy and 3D retinal reconstruction}

All confocal images were acquired with a Leica TCS SP8 confocal equipped with a tunable white light laser, and processed using Photoshop (Adobe). Images were assembled using the LASX software (Leica). 3D structures were constructed using the "Blend" volume representation of the LASX software. The depth color coding was used to represent better the depth of the layers, coloring in warm colors the superficial plexus, and colder colors the deeper ones.

\section{Quantifications}

For quantifications, images were analyzed using ImageJ software (NIH).

For samples from Adgrf5-mCherry mice, the fluorescence intensity was calculated for each vascular bed proximal to an artery or a vein, respectively (within a $100 \mu \mathrm{m}$ distance), and averaged for three retinae per age.

For vessels extending from the superficial to the inner plexus, vessels were quantified on whole retinas $3 \mathrm{D}$ pictures 
along each full artery or vein, using the first capillary bifurcation as the lateral limit. Three retinae were used per genotype.

For inner plexus densities, the vascular density plugin was used on $500 \mu \mathrm{m}^{2}$ areas inside the inner plexus of three retinae per genotype. To further characterize the capillary organization, the Analyze particles plugin was used and the resulting perimeters of the vascular structures distributed by size ( 0 to $10 \mu \mathrm{m}, 10$ to $100 \mu \mathrm{m}, 100$ to $1000 \mu \mathrm{m}$, over $1000 \mu \mathrm{m}$ ).

\section{mRNA extraction and quantification}

Whole snap-frozen organs were homogenized using the FastPrep-24 (Bertin) machine. Total RNA was extracted with RNeasy Mini Kit (QIAGEN) following the manufacturer's protocol. The RNA quantity was measured on a Synergy HT Microplate machine (BioTek). The RNA quality was determined by the ratio of absorption A260/A280, and was considered as acceptable in the range of 1.9-2.1.

\section{Quantitative RT-PCR on organs and EC}

cDNA was synthesized from $0.5 \mu \mathrm{g}$ total RNA using the iScript cDNA Synthesis Kit (Bio-Rad). Real-Time quantitative PCR (qRT-PCR) was performed using TaqMan® Gene Expression Master Mix (Life technologies) on a CFX96 Touch $^{\mathrm{TM}}$ Real-Time PCR Detection System (Bio-Rad). The expression level was normalized to Hprt as a housekeeping gene.

The following probes were used: mouse Adgrf5 (Assay: Mm01269028_m1, FAM, spanning exon 17-18, Life Technologies), Pecam-1 (Assay: Mm01242576_m1, FAM, Life Technologies), Hprt (Assay: Mm03024075_m1, FAM, Life Technologies).

\section{Statistical analysis}

Data are expressed as mean $\pm \mathrm{SD}$. In datasets containing two distinct groups, statistical comparisons were performed with the Student's $t$ test, and $p<0.05$ was considered statistically significant. In dataset containing three distinct groups, statistical comparisons among groups were performed using one-way ANOVA followed by Tukey's post hoc test and $p<0.05$ was considered statistically significant. On the figures, the error bars represent $\mathrm{SD}$, and $p<0.05$ is represented as $* p<0.005$ as $* * p<0.0005$ as $* * * p<0.00005$ as $* * * *$ and "ns" stands for "no significant difference."

\section{Results}

\section{The inner plexus develops coaxially to the overarching veins}

Using a transgenic reporter mouse line that labels EC, Cldn5-GFP, we first studied the physiological progression of retinal vascularization in otherwise WT mice. We observed that the inner plexus develops following a stereotypical pattern during mouse retinal development. In a C57/B16 background, the first sprouts that emerge from the superficial plexus started to form the inner plexus around $\mathrm{P} 8$, which reached the retinal periphery at $\mathrm{P} 9$ and covered the entire retinal surface at P10 (Fig. 2a, "Superficial plexus"). Furthermore, we found that the inner plexus emerged below the veins and surrounding capillaries of the superficial plexus at the initial stage (P8), and these initial vascular patches became denser at later stages ( $\mathrm{P} 9$ and P10, Fig. 2a, "Inner plexus"), while the rest of the inner plexus kept extending radially.

To understand this coaxial progression, we tracked the vascular sprouts diving from the superficial plexus that form the inner vascular network at P8. Numerous vertical sprouts were detected below the perivenous area, in contrast to the arterial region (Fig. 2b, "3D vertical view"). The perivenous sprouts showed a highly developed and complex pattern, as revealed by immunofluorescence staining with the luminal marker, ICAM2 (Fig. 2b, "3D vertical view"). This coincided with a dense capillary network around the veins in the superficial plexus, as compared to the sparse capillary network surrounding the artery (Fig. 2b, "Superficial plexus").

Once initiated at P8, the numerous vertical vascular projections and the resulting patches of high density in the inner plexus below the veins (Fig. 2c, "3D vertical view" and "Inner plexus") were still observed while the inner plexus developed at P9 and P10 (Fig. 2c, Inner plexus). Meanwhile, the perivenous capillary network in the superficial plexus was progressively normalized (Fig. 2c, "Superficial plexus").

We tested whether active EC migration is required to prolong the high EC density in the perivenous area. Rac1 is a Rho GTPase critical for cell migration [40]. Therefore Racl gene was abrogated postnatally, using conditional endothelial-specific Racl knockout mice (Racl ECKO). In mutants, the number of sprouts derived from the perivenous area that reached the inner plexus was reduced at P8 (Fig. 2d, "Inner plexus" and "3D vertical view," quantified in Fig. 2e). However, the overarching superficial network exhibited an unaltered density (Fig. 2d, "Superficial plexus"). These results suggest that the inner plexus formation requires EC endowed with intact Rac 1 activity in contrast to the formation of the superficial plexus, which develops independently of Rac1. 

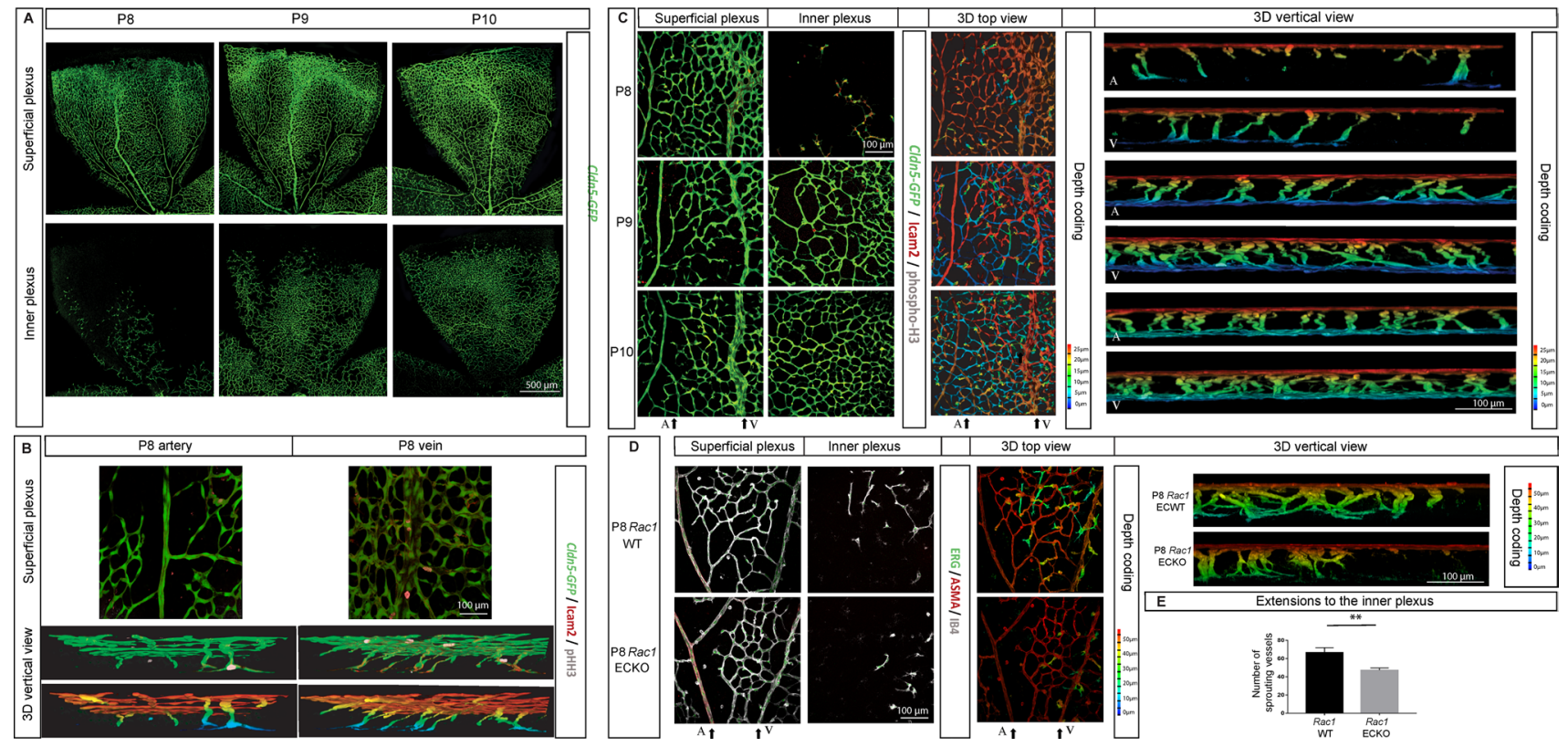

Fig. 2 Retinal vascular inner plexus develops coaxially to the overarching veins. a Morphology of the superficial (upper) and inner (lower) vascular plexus from Cldn5-GFP transgenic reporter mouse retina at $\mathrm{P} 8, \mathrm{P} 9$, and $\mathrm{P} 10$. The vascular network is genetically labeled (GFP, shown in green). "A" and "V" indicate the position of an artery and a vein, respectively. b Visualization of the superficial vascular layer around an artery (left) and a vein (right) of Cldn5-GFP mouse retina at $\mathrm{P} 8$. The EC projections toward the inner plexus specific to each area were reconstructed (3D vertical view). Retinal EC were genetically labeled by Cldn5-GFP (green) and further stained for ICAM2 (red) and phospho-histone $\mathrm{H} 3$ (pHH3, gray) to visualize vas-

\section{Adgrf5 is exclusively endothelial in the retina}

We and others have shown that Adgrf5 is expressed in the endothelium of all vascular beds of major organs in mice, though non-EC cell types might also express it in some tissues $[28,31]$. In the specific case of the retinal vasculature, we first measured the mRNA expression of Adgrf5 at various stages of development by real-time PCR. In C57/B16 animals, Adgrf5 and the endothelial marker Pecam- 1 revealed a similar expression pattern with a nearly twofold increase over the first week of postnatal development followed by a threefold, and a fivefold decrease up to the third month of development for Adgrf5 $(0.15 \pm 0.02, p \leq 0.0001)$ and Pecam-1 (0.09 $\pm 0.02, p \leq 0.0001)$, respectively (Fig. 3a). These results indicate that the mRNA level of Adgrf5 is strongly correlated with the expression of the endothelial marker Pecam-1. Overall, the ratio of Adgrf5 to Pecam-1 transcripts showed a steady profile from $\mathrm{P} 1$ to $\mathrm{P} 14$ followed by a 1.7 -fold increase $(0.9 \pm 0.4$ to $1.62 \pm 0.17, p=0.0002)$ from P7 to P14, and plateaued again during adulthood (Fig. 3b).

To determine whether the Adgrf5 gene is exclusively expressed by EC in the postnatal retina, we generated a cular lumen and proliferation, respectively. Depth coding of the same area is shown. c Superficial (left), inner (middle left) vascular plexus, and 3D overlay view from top (middle right) of Cldn5-GFP mouse retina at $\mathrm{P} 8, \mathrm{P} 9$, and $\mathrm{P} 10$. 3D vertical view below the artery or the vein is shown (right). d Superficial (left), inner (middle left) vascular plexus, and 3D top view (middle right) of WT and Racl ECKO retina. A vertical view below the vein $(\mathrm{V})$ is shown (right). Artery (A) and vein $(\mathrm{V})$ are indicated. e Quantification of the EC projections to the inner plexus observed below the vein in Racl ECKO and WT mouse retina at $\mathrm{P} 8$

constitutive, endothelial-specific deletion mouse model of ADGRF5 ("Adgrf5 ECKO"), by crossing a mouse carrying a floxed Adgrf5 exon 17 to a Tie2-Cre mouse line [41]. Our real-time PCR data revealed that the Adgrf5 mRNA level in Adgrf5 ECKO mouse retina $(0.006 \pm 0.004)$ was reduced to $2.4 \%$ of the WT littermates $(0.244 \pm 0.06, p \leq 0.0001)$ at P7. At P21, Adgrf5 ECKO mice showed $0.5 \%$ of the Adgrf5 transcript levels compared to littermate control retina $(0.001 \pm 0.0001$ for Adgrf5 ECKO, $0.196 \pm 0.04$ for $\mathrm{WT}, p \leq 0.0001$ ) (Fig. 3c). These results suggest that, upon genetic ablation of Adgrf5 in the endothelium, the expression level of Adgrf5 in the retina is completely abrogated, hence excluding expression in other cell types than EC.

Next, we employed a complementary approach to confirm the endothelium-specific expression of Adgrf5, and analyzed the retinal vasculature of the Gpr116(Adgrf5)mCherry mice, which express mCherry under the control of the Adgrf5 promoter and its enhancer elements [38]. Expression of Adgrf5 co-localized with the isolectin B4 (IB4)-positive endothelium in the superficial plexus at $\mathrm{P} 9$, indicating EC-specific ADGRF5 expression in the retinal vasculature. Starting at P14, Adgrf5 expression in the inner plexus was also observed. The strong expression pattern of Adgrf5 in 

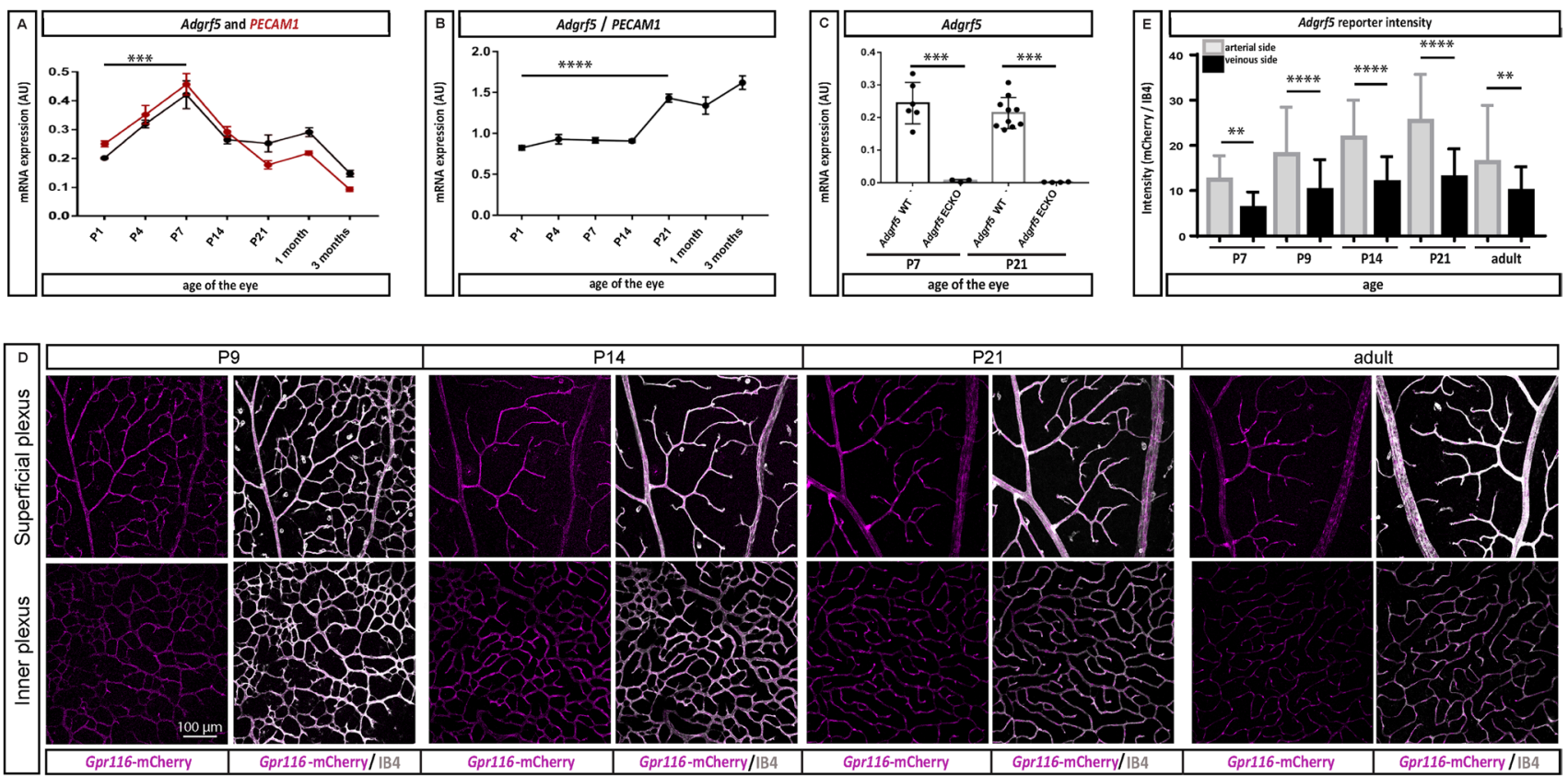

Fig. 3 Adgrf5 is exclusively endothelial in postnatal retina. a mRNA expression levels of Adgrf5 (black line) and Pecam-1 (red line) in mouse retina. The whole retinal RNA from C57BL6 mice was used to determine Adgrf5 and Pecam-1 transcripts by qRT-PCR at P1 $(n=6$ mice), P4 ( $n=4), \mathrm{P} 7(n=3), \mathrm{P} 14(n=5), \mathrm{P} 21(n=5), 1$ month $(n=3)$, and 3 months $(n=4)$. The expression of each gene was normalized by Hprt. Error bars represent \pm SD. b Relative mRNA expression level of Adgrf5 normalized by Pecam-1 in C57/B16 retinae. The same number of animals mentioned in Fig. 2a was used. c Adgrf5 mRNA expression level over the course of the eye development. Adgrf5 WT and Adgrf5 ECKO mouse retinal RNA was prepared at the time indi-

the superficial plexus was maintained over the course of the retinal vasculature development (Fig. 3d). Specifically, the highest reporter activity was observed on the arteriole side and branching points within the superficial plexus (Fig. 3e). Reporter expression was further detected in the horizontal cells below the outer nuclear layer in 3-month-old animals (data not shown).

\section{Adgrf5 deficiency leads to increased vascular density around the veins in retina}

The expression pattern of Adgrf5 in the superficial plexus prompted us to re-examine retinal vasculature in Adgrf5deficient mice. We previously showed that Adgrf5 KO retinal vasculature did not exhibit any obvious growth defect at P4. However, P9 retina from Adgrf5 KO animals revealed a denser retinal capillary network around the vein as well as an increased number of inner sprouts detected below the perivenous area, and a more developed inner plexus (Fig. 4a, "Superficial plexus" and "Inner plexus"). These results suggest that the increased inner sprouts observed in Adgrf5 KO mice (Fig. 4b) might have accelerated the cated and Adgrf5 transcript was assessed by qRT-PCR (Adgrf5 WT $(n=6)$ and Adgrf5 ECKO $(n=3)$ at P7 and Adgrf5 WT $(n=10)$ and Adgrf5 ECKO $(n=4)$ at P21). Results are normalized by Hprt expression. d Fluorescent images of ADGRF5 expression in-between an artery and a vein in superficial (upper) and inner (lower) vascular layer. Gpr116-mCherry reporter mouse retinae were collected at the time indicated, and stained with IB4 (gray) to visualize the endothelium. mCherry indicates Adgrf5 expression (magenta). e Quantification of the fluorescence intensity in Gpr116-mCherry reporter mouse retinae, normalized over isolectin (lB4) fluorescence

formation of a denser inner network. Additionally, Adgrf5 ECKO mice showed a similar pattern as Adgrf5 KO, specifically an increase of the vascular density around veins and arteries in the superficial plexus (Fig. 4c, "Superficial plexus") and a higher number of inner sprouts (Fig. 4d). We found that, once the sprouts formed the inner plexus, it became denser and more complex in mutants than in littermate WT (Fig. 4c, "Inner plexus"). These data indicate that endothelial ADGRF5 is involved in the regulation of the perivenous capillary density, which subsequently influences inner plexus formation.

As genetic ablation of Adgrf5 in mice causes a denser vasculature, both in the superficial and in the inner plexus, we addressed the effect of Adgrf5 overexpression. To this end, we generated a gain-of-function Adgrf5 mouse model. Specifically, Adgrf5 gene was overexpressed in the endothelium by crossing the $\mathrm{Gt}($ Rosa26-CAG-Adgrf5) mouse to a Tie2Cre line. These mice, named Adgrf5 endothelial cell gainof-function (Adgrf5 ECGOF), showed a sparse perivenous network in the retinal vasculature at P9 (Fig. 4e). Moreover, a low number of endothelial sprouts protruded through the inner nuclear layer (INL) (Fig. 4f), and the inner plexus was 

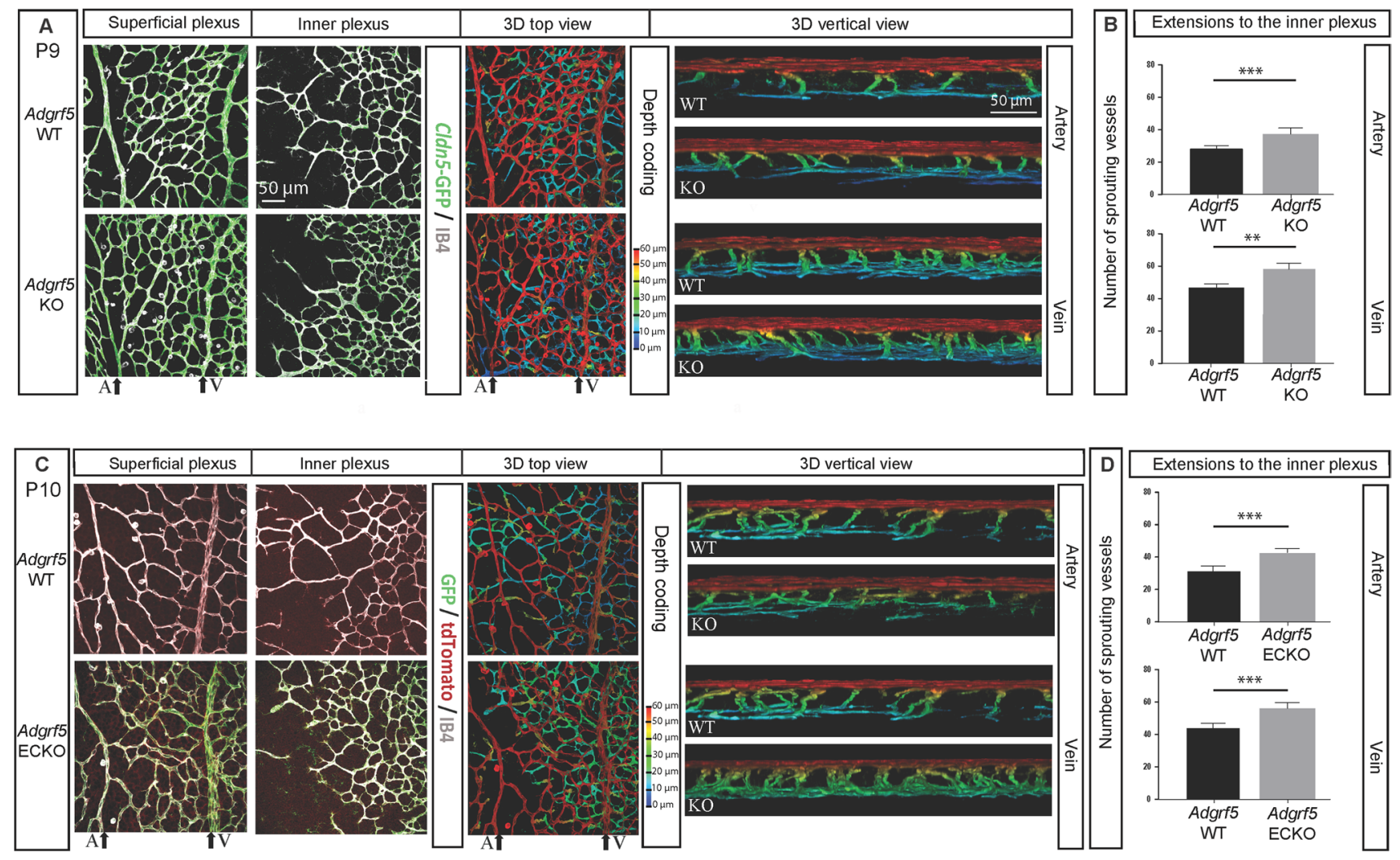

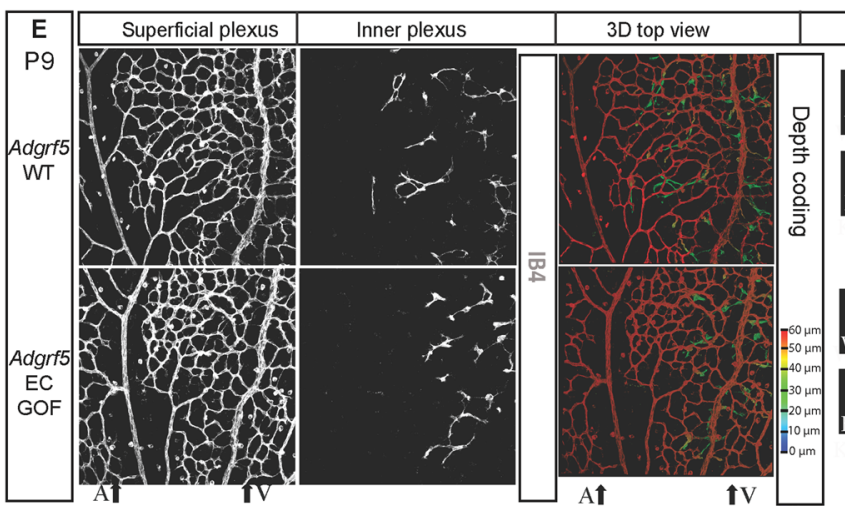

Fig. 4 Adgrf5 deficiency leads to perivenous abnormalities. a Involvement of Adgrf5 in the inner plexus formation. Superficial (left) and inner (middle left) vascular plexus in-between an artery and a vein from WT (upper) and Adgrf5 KO (lower) retina at P9 are shown. The endothelium is visualized by Cldn5-GFP reporter (green) and IB4 staining (gray). Complete 3D reconstruction of the same area is represented in depth coding with a top view (middle), and a vertical view below the vein $(\mathrm{V})$ and an artery $(\mathrm{A})$ is indicated (right panel). b Quantification of the number of extensions from the superficial to the inner vascular layer in WT and Adgrf5 KO under the arteries (top) and under the veins (bottom). c Role of EC-specific Adgrf5 in the inner plexus development. Superficial (left) and inner (middle left) vascular layer in-between an artery and a vein in the WT (upper) and Adgrf5 ECKO (lower) retina at $\mathrm{P} 10$ are shown. The endothelium is visualized by IB4 staining (gray). Adgrf5-depleted EC are marked by GFP (green). Complete 3D reconstruction of the area from WT and
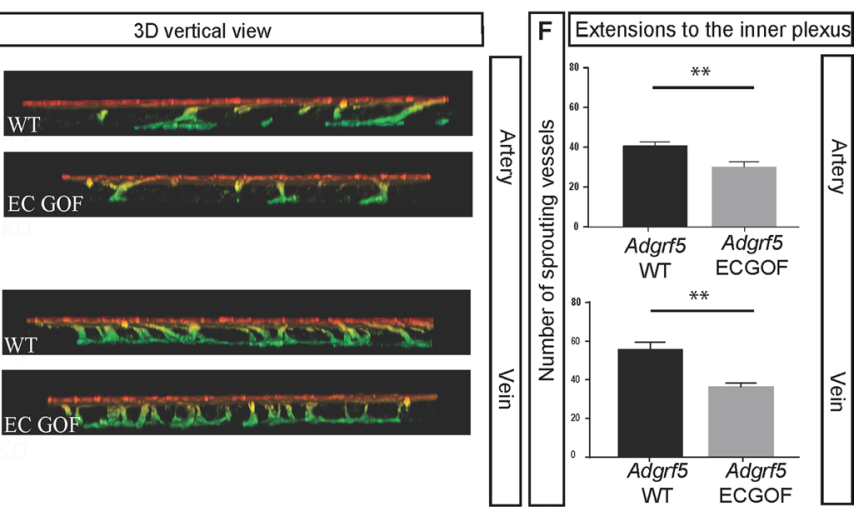

Adgrf5 ECKO is represented in depth coding with a top view (middle), and a vertical view below the vein (V) and an artery (A) is indicated (right panel). d Quantification of the EC protrusions from the superficial to the inner vascular layer in WT and Adgrf5 ECKO under the arteries (top) and under the veins (bottom). e Role of EC-specific Adgrf5 in the inner plexus development. Superficial (left) and inner (middle left) vascular layer in-between an artery and a vein in the WT (upper) and Adgrf5 ECGOF (lower) retina at P9 are shown. The endothelium is visualized by IB4 staining (gray). Complete 3D reconstruction of the area from WT and Adgrf5 ECGOF is represented in depth coding with a top view (middle), and a vertical view below the vein (V) and an artery (A) is indicated (right panel). f Quantification of the EC protrusions from the superficial to the inner vascular layer in WT and Adgrf5 ECGOF under the arteries (top) and under the veins (bottom) 
sparse compared to WT littermates. Taken together, these data suggest that the level of endothelial ADGFR5 is critical for regulating the perivenous vascular density in the superficial plexus, thus tightly controlling the inner plexus density.

\section{Loss of ADGRF5 triggers subretinal neovascularization}

In order to explore the impact of ADGRF5-deficiency on the inner plexus morphology, we performed a longitudinal study. In the Cldn5-GFP WT mouse retina, the inner plexus that is initially composed of equal-sized and dense capillaries at P12 undergoes a progressive remodeling, which leads to the appearance of higher caliber vessels at P14. The inner network remodeled around those bigger vessels, becoming sparse and regular in the juvenile mice at P21 and 1 month of age, and this feature was further preserved over adulthood (Fig. 5a, top row). Interestingly, during the whole process, the inner plexus of WT mice was devoid of any deep sprouts that would dive into the outer nuclear layer (Fig. 5a, lower row). The inner plexus of Adgrf5 KO retina, on the other hand, showed patches of capillaries with increased vascular density at P12 and P14, which were progressively normalized over age (Fig. 5b, quantified in Fig. 5d, e). Indeed, Adgrf5 KO inner retinal layer was virtually indistinguishable from WT control group at 3 months (Fig. 5b, top row).

Vascular growth and maturation is accompanied by basement membrane deposition. The first sprouts reaching the inner plexus deposited very low amount of collagen IV (P14, Fig. 5c). As the inner plexus develops, the intensity of collagen IV-positive staining increased (P21, Fig. 5c) until it became very high in the mature vascular network (1 month, Fig. 5c). This increase in collagen IV deposition was delayed until P21 in the Adgrf5 KO retinae, despite the extensive vascular development of the inner plexus (IB4-positive), implying a delay in maturation both at the morphogenetic and the basement membrane levels (Fig. 5c).

We noticed that the clusters of capillaries with high density in the inner plexus in the Adgrf5 KO mouse retina coincided with sprouts emanating from the deep plexus toward the subretinal space at P14 (Fig. 5b, lower row, quantified in Fig. 5f, g). This led us to check where these cells originated from. By analyzing the endothelial expression pattern of Cldn5-GFP and 1B4-positive cells, we found that these inner sprouts arose from the pre-existing capillaries forming the deep plexus. Additionally, the perivenous coalescent superficial network, the corresponding denser capillaries of the inner plexus, and the region of origin of the inner sprouts showed a close spatial correlation (Fig. $5 \mathrm{~h}$ ). We found that the more developed sprouts in the inner retinal space were closely associated with vascular clusters of very high density in the superficial plexus (Fig. 5h, Adgrf5 KO (severe)).
Since the Adgrf5 KO mice were generated by a large excision of the gene [28], spanning exon 3 to exon 21, a potential off-target effect due to either a deleted intronic sequence or the C57/B16 background could occur. To exclude these possibilities, we generated another mouse model harboring a mutation in exon 17 from the Adgrf5 gene. This exon encodes for the seven transmembrane domains. Specifically, germ line deletion was achieved by crossing a male carrying the floxed exon 17 of the Adgrf5 gene to a Tie2-Cre-positive female, since the activation of Cre recombinase by the Tie 2 promoter occurs during oocytogenesis. Thus, the floxed gene will be removed in zygote, ultimately resulting in global deletion of the gene [41]. The efficiency of the LoxP/Cre recombination in offspring was examined by PCR to select mutants that have lost exon 17 (Supplementary Figure 1a). Interbreeding of the offspring produced mutants and heterozygotes, which showed a complete or a half silencing of Adgrf5 expression, respectively (Supplementary Figure 1b). This suggested instability of the mRNA after exon 17 excision. Next, we verified that this strain, called Adrgf5 $\Delta 17$, can recapitulate the lung defects previously described in the Adgrf5 KO [29-31]. Indeed, the Adrgf5 $\Delta 17$ lung showed increased foamy cells and alveolar enlargement at 3 weeks of age (Supplementary Figure 1c). Moreover, 2-monthold Adrgf5 $\Delta 17$ mice failed to retain a $1 \mathrm{kDa}$ Alexa Fluor 555-cadaverine tracer in the CNS blood vessels, leading to tracer accumulation in the brain parenchyma (Supplementary Figure 1d). Overall, Adrgf5 $\Delta 17$ mice recapitulated the main phenotypical abnormalities previously demonstrated in Adgrf5 KO.

At P14, Adrgf5 $\Delta 17$ retina exhibited a marked sprouting from the deep plexus toward the subretinal space compared to WT littermates (Fig. 5i, P14 Adgrf5 $\Delta 17$ ), indicating that this phenotype is neither caused by the genetic background nor a neighboring effect in the full gene deletion model. Additionally, we examined Adgrf5 ECKO mouse retina at P14 and observed sprouts diving from the deep plexus, confirming that the subretinal neovascularization phenotype observed upon loss of Adgrf5 was endothelial-driven (Fig. 5j, P14 Adgrf5 ECKO). Conversely, Adgrf5 ECGOF retina showed no signs of dense EC clusters in the inner plexus, nor any subretinal sprouting at P14 (Fig. 5k).

Further histological characterization of the subretinal sprouts in Adgrf5 $\mathrm{KO}$ using cross-sectioned retina revealed the presence of lesions descending from the OPL inside the ONL. The lesions were occasionally surrounded by erythrocytes, implying a possible defect in blood-retinal barrier establishment (Fig. 6a, H\&E staining and Fig. 6b). Tracking double Cldn5-GFP- and IB4-positive labeling in retinal sections of the Adgrf5 KO mice confirmed that neovessels penetrated the ONL, extending approximately $50 \mu \mathrm{m}$ into the subretinal space, but seldom reached the outer segment (Fig. 6a, right column). These vascular lesions are 

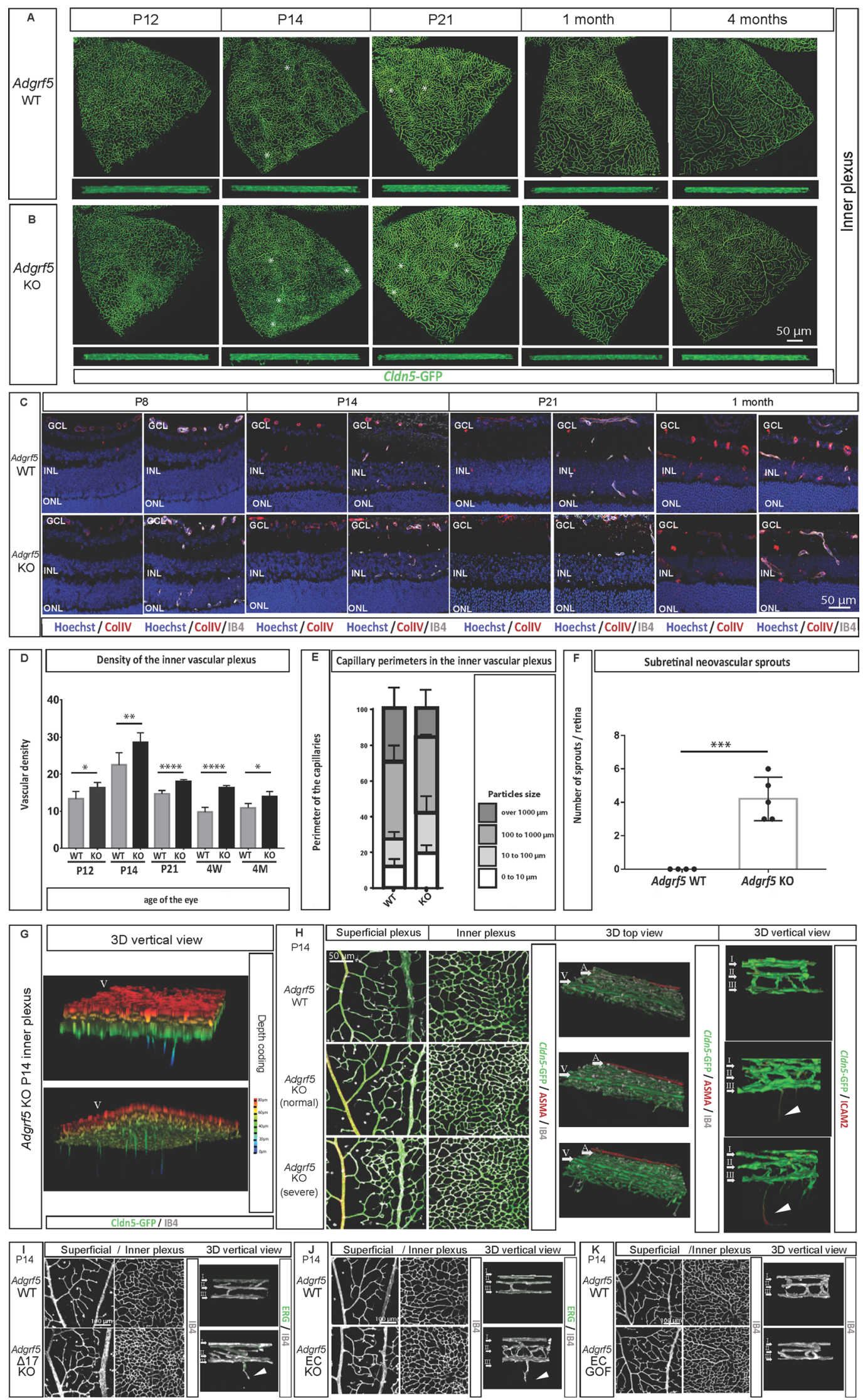
4Fig. 5 Adgrf5 KO retina develops transient neovessels in the inner retinal space. a Inner vascular layer in a leaf of WT mouse retina at $\mathrm{P} 12, \mathrm{P} 14, \mathrm{P} 21,1$ month, and 4 months. The endothelium is visualized in green tracking Claudin5-GFP. Horizontal view (upper panel) and optical cross-section (lower panel) are presented. Asterisks indicate areas of high vascular densities. b Inner vascular layer in a leaf of Adgrf5 KO mouse retina at P12, P14, P21, 1 month, and 4 months. The endothelium is visualized in green tracking Claudin5GFP. Horizontal view (upper panel) and optical cross-section (lower panel) are presented. c Images from Adgrf5 WT (top row) and Adgrf5 KO (lower row) mouse retina at P8, P14, P21, and 1 month. Crosssectioned retinae were stained with collagen IV (red) and Hoechst (blue). The vasculature is depicted by IB4 staining (gray). d Quantification of the vascular densities in the inner plexus from Adgrf5 WT or KO mouse retina at P12, P14, P21, and 1 month. e Quantification of the capillary perimeters in the inner plexus from Adgrf5 WT or KO mouse retina at P14. f Quantification of the subretinal neovascular sprouts emerging from the inner plexus in Adgrf5 WT or KO whole mouse retina at $\mathrm{P} 14$. g $3 \mathrm{D}$ reconstruction images of the three vascular layers below the vein of Adgrf5 $\mathrm{KO}$ retina at P14. The retinal structure is presented in depth coding, displaying EC extensions after the inner vascular layer in the subretinal space (deep sprout is marked by an arrowhead). h Superficial (left) and inner (middle left) retinal vascular layer in-between an artery and a vein in WT and Adgrf5 KO (normal and severe) mice at P14. The 3D reconstruction images of the three vascular layers and the neovessels below (middle right) are shown. IB4 (gray) and Cldn5-GFP (green) were used to label the endothelium, followed by ASMA staining to distinguish the artery and the vein. Vertical 3D projection of the three vascular layers and the neovessels below in the Adgrf5 $\mathrm{KO}$ at high magnification ( $\times 63)$ (right) (deep sprout is marked by an arrowhead). i Superficial (left), inner (middle) vascular plexus and 3D view (right) from WT and Adgrf5 $\Delta 17 \mathrm{KO}$ at P14. The vascular layers were stained with IB4 (gray) (deep sprout is marked by an arrowhead). j Superficial (left), inner (middle) vascular plexus and 3D view (right) from WT and Adgrf5 ECKO at P14. The vascular layers were stained with IB4 (gray) (deep sprout is marked by an arrowhead). k Superficial (left), inner (middle) vascular plexus and 3D view (right) from WT and Adgrf5 ECGOF at P14. The vascular layers were stained with IB4 (gray) (deep sprout is marked by an arrowhead)

reminiscent both in their sequence and histological nature of the early steps of RAP, where EC proliferation in the inner plexus precedes sprouts toward the subretinal space. They were not detected after P21 (Fig. 6c), and did not seem to anastomose with the choroidal vascular system (Supplementary Figure 2).

We investigated whether Adgrf5 deficiency would result in retinal tissue damages around the vascular lesions. Besides the subretinal hemorrhages around the neovessels in the ONL (Fig. 6a), Adgrf5 KO mouse retina displayed a disorganized GCL at P14 (NeuN staining, Fig. 6c). In agreement with the transient nature of the vascular lesions, the ganglion cells at later stages seemed intact. Glutamine synthetase staining results showed a normal development of Müller cells, which extended progressively from the GCL toward the inner plexiform layer, without any changes in the areas of subretinal neovascularization (Fig. 6d). GFAP staining, usually upregulated in situations of retinal stress, was confined to the GCL layer, confirming that the retinal damages were relatively limited
(Fig. 6e). These results indicate that retinal gliosis did not occur in Adgrf5 KO.

Overall, in line with the vascular normalization of the inner plexus over time in Adgrf5 KO retinae, ADGRF5 deficiency does not cause any permanent histological abnormalities in adult mouse retina, suggesting a progressive recovery.

\section{Discussion}

\section{Adgrf5 deficiency results in abnormal retina patterning}

This study extends our understanding of ADGRF5 function in patterning of the retinal vasculature. Adgrf5 is strongly expressed in EC of different vascular beds, especially in the CNS endothelium. In fact, in the retina, only vascular endothelium seems to express Adgrf5. Yet Adgrf5 loss-of-function was never linked to any obvious defect in the vascular architecture of any organ [28]. Although a severe impairment in the aortic arch arteries and the cardiac outflow tract formation was reported using a double Adgrf5/Adgrl4 knockout mice, the phenotype did not seem to stem from an EC autonomous effect [38]. Here, we have shown that constitutive and endothelial-specific genetic ablation of Adgrf5 leads to a transiently increased perivenous vascular density in the developing postnatal retina. These findings support for the first time the connection between an endothelial-specific loss of Adgrf5 and a morphogenetic disorder that affects the vasculature.

The perivenous area is the latest region inside the superficial plexus undergoing vascular remodeling. Vascular remodeling encompasses multiple cellular events, such as cessation of proliferation, vascular pruning, and cell death, enabling nascent vessels to become fully functional. These events reshape the initial dense plexus into a sparser network, mostly from P10 to P14, starting in the vicinity of the artery and extending progressively toward the veins. Oxygen delivery, blood flow, and local cues are likely to mediate these events, although the balance between each of them remains poorly defined, especially along the artery-to-vein axis [42]. The cause of the incomplete vascular remodeling around the veins in Adgrf5 $\mathrm{KO}$ retinae requires further exploration to determine whether this is due to altered blood flow, a pressure-related systemic dysregulation or an impaired local mechanism. The former hypothesis is supported by the predominant expression of Adgrf5 on the arteriole side, whereas the defect seems to localize in the perivenous plexus (Figs. 3, 4). An impaired local mechanism would be in line with increasing evidence that aGPCRs can act as mechanosensors [43, 44]. 

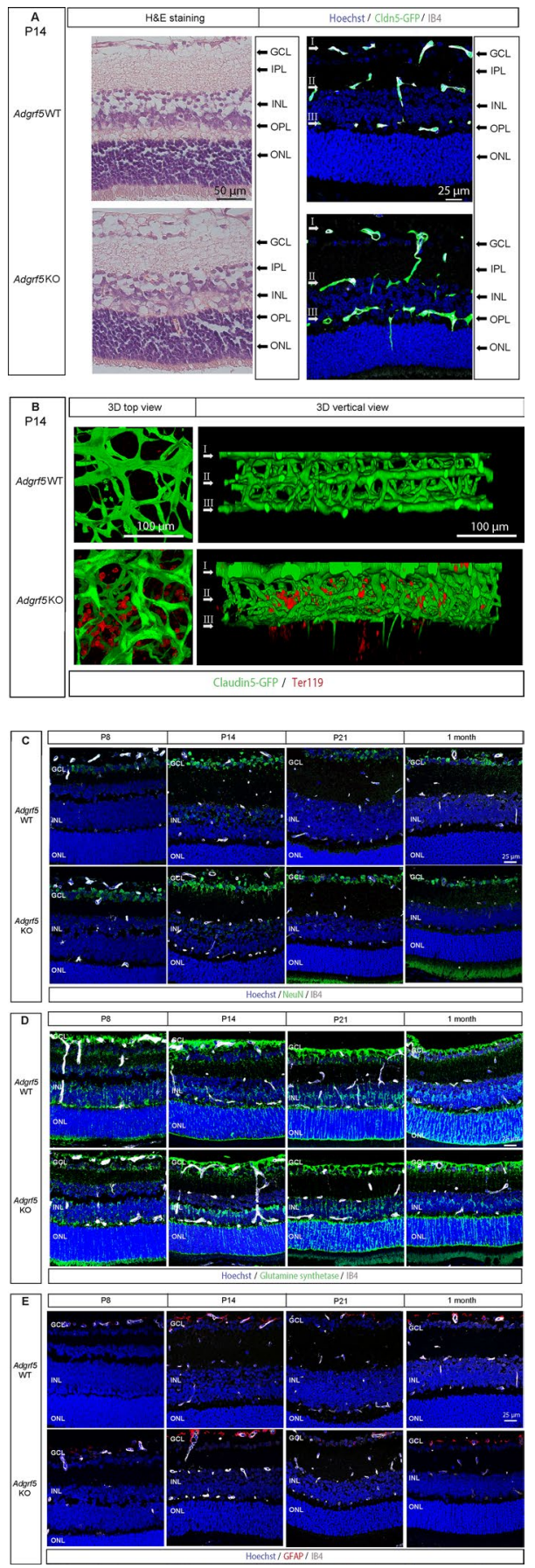

Fig. 6 Adgrf5 KO retinal tissue recovers after vessel normalization. a Images from WT and Adgrf5 KO mouse retina at P14. Cross-sectioned retinae were stained with H\&E (top) and Hoechst (blue). The vasculature is depicted by IB4 staining (gray) and Claudin5-GFP expression (green). b 3D reconstruction images of the three vascular layers of Adgrf5 KO retina at P14. The retinal structure is presented viewed from the superficial layer (3D top view, left), or from a vertical view (right). Erythrocytes are visualized by Ter119 staining. $\mathbf{c}$ Images from WT and Adgrf5 $\mathrm{KO}$ mouse retina at P8, P14, P21, and 1 month. Cross-sectioned retinae were stained with anti-NeuN antibody (green), Hoechst (blue), and IB4 (gray). d Images from WT and Adgrf5 KO mouse retina at P8, $\mathrm{P} 14, \mathrm{P} 21$, and 1 month. Cross-sectioned retinae were stained with anti-Glutamine synthetase antibody (green), Hoechst (blue), and IB4 (gray). e Images from WT and Adgrf5 KO mouse retina at P8, P14, P21, and 1 month. Crosssectioned retinae were stained with anti-GFAP antibody (red), Hoechst (blue), and IB4 (gray)

\section{Perivenous endothelial cells link the superficial and the inner layer in developing retinal vasculature}

We interrogated the role of the perivenous area during deep plexus formation. We have found that superficial and inner plexus are physically connected by forming vascular bridges in-between the two plexi at $\mathrm{P} 9$, as evidenced by using the endothelial Cldn5-GFP reporter mouse model. We also showed that vascular sprouts radiating from the perivenous region of the superficial plexus initiate the inner plexus formation (Figs. 2a-c). This process requires active EC migration, as demonstrated by genetic deletion of Rac1, a small Rho GTPase essential for cell migration in vivo [40]. Consequently, the absence of Rac1 significantly impinged on the progression of the perivenous sprouts toward the inner plexus (Fig. 2d). Our results suggest that Rac1 is not critical for Adgrf5 retinal phenotype. Although ADGRF5 has been suggested to be coupled to RhoA and Rac1 in vitro [45], Racl ECKO retinae revealed normal perivenous densities but severely reduced projections to the inner plexus, which was clearly different from the phenotype in Adgrf5 $\mathrm{KO}$ mouse retinae displayed in Fig. 2d and [46].

To decipher the role of the superficial vascular plexus during the initial steps of inner plexus development, we have used Adgrf5-deficient mice as a genetic model to alter perivenous vascular density in the developing retina. As a consequence of the high perivenous vascular density in Adgrf5 KO retinae, we observed an increased projection of vascular sprouts from these vessels toward the INL. This resulted in accelerated formation of the inner vascular plexus (P9) with very dense capillary patches below the vein (P14). These results suggest that the capillaries descending from the perivenous area are the main contributors to the inner plexus. Thus, an increased density in the superficial plexus in the perivenous area would consequently lead to inner plexus abnormalities. Yet, the pathways that control sprouts diving remain to be explored as most vascular guidance cues discovered to this point can modulate the bifurcation leading to new plexus formation $[4,5]$. In particular, perivenousderived sprouts could be endowed with a specific programing to dive into the retinal space, or alternatively the subvenous area might be delineated by specific guidance cues.

\section{Superficial perivenous abnormalities can lead to transient vascular projections into the neuroretina}

We observed that the abnormal clusters of capillaries observed in the inner plexus of Adgrf5 KO retinae evolved into vascular projections inside the inner retinal space. RAP is a sub-class of pathological retinal neovascularization syndrome, characterized by neovessel growth from the 
inner plexus into the neuroretina [9]. Subretinal vascularization is usually defined as the second step toward a full RAP syndrome [10]. In most murine models of subretinal neovascularization, endothelial projections are detected around 2 weeks after birth. This coincides with massive developmental vascular remodeling of the inner vascular plexus, which at that time consists of very dense and rather randomly organized capillary networks. Our data suggest that this immature stage of the inner plexus development is strongly influenced by the overarching superficial plexus.

The mouse models currently available to examine subretinal vascularization are based on mutations that affect cell types distinct from the endothelium and disrupt the architecture of the neuroretina $[47,48]$, or that render the photoreceptor layer metabolically deprived, as established in the well-studied Vldr KO mouse model [15]. These findings demonstrated that alterations of the neuroretina, triggering cell-to-cell communication with the endothelium could eventually cause a burst of growth factors which influences the vascular invasion of the inner retina. Furthermore, the status of the endothelium using these mouse models has not been characterized in depth.

\section{Vascular invasion of the neuroretina as a function of the endothelium status}

Intrinsic properties of the retinal endothelium might also have an impact on the RAP process. The first evidence for an important role of the endothelium was brought by the longitudinal study of mice carrying a vascular-specific, dominant-negative mutation in collagen type IV, alpha 1 (COL4A1) [16]. Col4a1 mutant retina developed vascular lesions arising from the deep vascular plexus and extending toward the RPE where they formed chorioretinal anastomoses. Our study supports the role of the retinal endothelium in establishing subretinal neovascularization. As shown in Fig. 3, Adgrf5 is exclusively expressed in the endothelium of the postnatal retinal vasculature. The Adgrf5 $\mathrm{KO}$ mouse model, therefore, can be useful as a new model to study endothelial-driven subretinal vascularization.

The exact origin of subretinal neovascularization has been debated after the formal identification of retinal angiomatous proliferation as a subset of lesions distinct from AMD [10, 49]. Together with Col4al mutants, our results from global and endothelial Adgrf5 KO mice reinforce the possibility that RAP lesions directly originate from disturbed retinal vascular beds, without starting at the choroidal level.

Our data demonstrate that the vascular maturation process can considerably impact the subretinal vascularization. We have shown that upon remodeling, the superficial plexus is highly permissive for projections toward the inner plexus. Moreover, our results suggest that the maturation process of the inner plexus might exert a second level of control over the subretinal neovascularization: from $\mathrm{P} 14$ to $\mathrm{P} 21$, the inner plexus remodeling occurs and higher caliber vessels develop surrounded by a sparse network [4]. Indeed, this strong remodeling of the inner plexus appeared to abolish the deep sprouts in Adgrf5 KO, and might represent a safeguard against subretinal neovascularization. In this regard, the expression of collagen IV, which was delayed in the inner plexus of Adgrf5 KO, became normalized around that time point. Given the severe abnormalities seen in mouse retinae carrying a dominant-negative Col4al mutation [16], it is possible that the basement membrane contains key maturation factors. The absence of any apparent histological alterations in adult Adgrf5 KO retina also reinforces the idea of a protective normalization process.

Lastly, RAP is an age-related syndrome, and is assumed to affect mostly fully mature and established retinal vasculature. In adult mice, pharmacological abrogation of the shield offered by photoreceptor-produced sFlt-1 resulted in fast development of RAP lesions [17]. This indicates that some pathways are permanently required to maintain the vascular privilege. On the other hand, in most spontaneous RAP mice models, the subretinal vascularization occurs in the vascular bed undergoing active remodeling at P14. It is unclear whether these transient pathways are involved and reactivated in late-onset pathologies. It would be interesting to investigate whether an equivalent "permissive stage" as the one observed in the immature inner plexus observed in mouse (at P14, for instance) exists in human.

In summary, we have shown that the endothelium status is important for triggering subretinal vascularization. We have illustrated how the degree of development and maturation of the retinal inner plexus controls the appearance of deep sprouts. Moreover, alteration of Adgrf5, an endothelialspecific gene in retina, plays a critical role in shaping the inner plexus by regulating a pool of perivenous EC in the superficial plexus. Activating ADGRF5 could therefore help preventing the development of RAP lesions.

Acknowledgements The authors thank Konstantin Gängel and Jennifer Hofmann for generating the Rac1 ECKO mice, Cecilia Olsson, Pia Peterson, Jana Chmielniakova, and Helen Leksell for technical assistance; Simon Stritt, Jennifer Hofmann, and Tobias Langenhan for insightful reviewing of the manuscript and the Biovis platform (http:// www.biovis.medfarm.uu.se/) for bright-field imaging. This study was supported by a grant from the European Research Council, AdG BBBARRIER.

Open Access This article is distributed under the terms of the Creative Commons Attribution 4.0 International License (http://creativeco mmons.org/licenses/by/4.0/), which permits unrestricted use, distribution, and reproduction in any medium, provided you give appropriate credit to the original author(s) and the source, provide a link to the Creative Commons license, and indicate if changes were made. 


\section{References}

1. Xia C, Lu E, Zeng J, Gong X (2013) Deletion of LRP5 in VLDLR knockout mice inhibits retinal neovascularization. PLoS ONE 8:e75186. https://doi.org/10.1371/journal.pone.0075186

2. Stahl A, Connor KM, Sapieha P, Chen J, Dennison RJ, Krah NM et al (2010) The mouse retina as an angiogenesis model. Invest Ophthalmol Vis Sci 51:2813-2826. https://doi.org/10.1167/ iovs. 10-5176

3. Selvam S, Kumar T, Fruttiger M (2018) Retinal vasculature development in health and disease. Prog Retin Eye Res. 63:1-19. https ://doi.org/10.1016/j.preteyeres.2017.11.001

4. Stefater JA, Lewkowich I, Rao S, Mariggi G, Carpenter AC, Burr AR et al (2011) Regulation of angiogenesis by a non-canonical Wnt-Flt1 pathway in myeloid cells. Nature 474:511-515. https:// doi.org/10.1038/nature10085

5. Wang Y, Rattner A, Zhou Y, Williams J, Smallwood PM, Nathans J (2012) Norrin/Frizzled4 signaling in retinal vascular development and blood brain barrier plasticity. Cell 151:1332-1344. https ://doi.org/10.1016/j.cell.2012.10.042

6. Ye X, Wang Y, Cahill H, Yu M, Badea TC, Smallwood PM et al (2009) Norrin, frizzled-4, and Lrp5 signaling in endothelial cells controls a genetic program for retinal vascularization. Cell 139:285-298. https://doi.org/10.1016/j.cell.2009.07.047

7. Okabe K, Kobayashi S, Yamada T, Kurihara T, Tai-Nagara I, Miyamoto $\mathrm{T}$ et al (2014) Neurons limit angiogenesis by titrating VEGF in retina. Cell 159:584-596. https://doi.org/10.1016/j. cell.2014.09.025

8. Lim LS, Mitchell P, Seddon JM, Holz FG, Wong TY (2012) Agerelated macular degeneration. Lancet (Lond Engl) 379:17281738. https://doi.org/10.1016/S0140-6736(12)60282-7

9. Jager RD, Mieler WF, Miller JW (2008) Age-related macular degeneration. N Engl J Med 358:2606-2617. https://doi. org/10.1056/NEJMra0801537

10. Yannuzzi LA, Negrão S, Iida T, Carvalho C, Rodriguez-Coleman $\mathrm{H}$, Slakter J et al (2001) Retinal angiomatous proliferation in agerelated macular degeneration. Retina Phila Pa. 21:416-434

11. Shimada H, Mori R, Arai K, Kawamura A, Yuzawa M (2005) Surgical excision of neovascularization in retinal angiomatous proliferation. Graefes Arch Clin Exp Ophthalmol Albrecht Von Graefes Arch Klin Exp Ophthalmol. 243:519-524. https://doi. org/10.1007/s00417-004-1073-x

12. Hasegawa E, Sweigard H, Husain D, Olivares AM, Chang B, Smith KE et al (2014) Characterization of a spontaneous retinal neovascular mouse model. PLoS ONE 9:e106507. https://doi. org/10.1371/journal.pone.0106507

13. Nagai N, von Leithner P, Izumi-Nagai K, Hosking B, Chang B, Hurd R et al (2014) Spontaneous CNV in a novel mutant mouse is associated with early VEGF-A-driven angiogenesis and late-stage focal edema, neural cell loss, and dysfunction. Invest Ophthalmol Vis Sci 55:3709-3719. https://doi.org/10.1167/iovs.14-13989

14. Hu W, Jiang A, Liang J, Meng H, Chang B, Gao H et al (2008) Expression of VLDLR in the retina and evolution of subretinal neovascularization in the knockout mouse model's retinal angiomatous proliferation. Invest Ophthalmol Vis Sci 49:407-415. https://doi.org/10.1167/iovs.07-0870

15. Joyal J-S, Sun Y, Gantner ML, Shao Z, Evans LP, Saba N et al (2016) Retinal lipid and glucose metabolism dictates angiogenesis through the lipid sensor Ffar1. Nat Med 22:439-445. https://doi. org/10.1038/nm.4059

16. Alavi MV, Mao M, Pawlikowski BT, Kvezereli M, Duncan JL, Libby RT et al (2016) Col4a1 mutations cause progressive retinal neovascular defects and retinopathy. Sci Rep 6:18602. https://doi. org/10.1038/srep18602
17. Luo L, Uehara H, Zhang X, Das SK, Olsen T, Holt D et al (2013) Photoreceptor avascular privilege is shielded by soluble VEGF receptor-1. eLife 2:e00324. https://doi.org/10.7554/elife.00324

18. Laviña B, Castro M, Niaudet C, Cruys B, Álvarez-Aznar A, Carmeliet $\mathrm{P}$ et al (2018) Defective endothelial cell migration in the absence of Cdc42 leads to capillary-venous malformations. Dev Camb Engl. https://doi.org/10.1242/dev.161182

19. Strutt D, Schnabel R, Fiedler F, Prömel S (2016) Adhesion GPCRs govern polarity of epithelia and cell migration. Handb Exp Pharmacol 234:249-274. https://doi.org/10.1007/978-3-319-41523 $-9 \_11$

20. Langenhan T, Prömel S, Mestek L, Esmaeili B, Waller-Evans H, Hennig C et al (2009) Latrophilin signaling links anterior-posterior tissue polarity and oriented cell divisions in the C. elegans embryo. Dev Cell 17:494-504. https://doi.org/10.1016/j.devce 1.2009.08.008

21. Usui T, Shima Y, Shimada Y, Hirano S, Burgess RW, Schwarz TL et al (1999) Flamingo, a seven-pass transmembrane cadherin, regulates planar cell polarity under the control of Frizzled. Cell 98:585-595

22. Chae J, Kim MJ, Goo JH, Collier S, Gubb D, Charlton J et al (1999) The Drosophila tissue polarity gene starry night encodes a member of the protocadherin family. Dev Camb Engl. 126:5421-5429

23. Luo R, Jeong S-J, Jin Z, Strokes N, Li S, Piao X (2011) G protein-coupled receptor 56 and collagen III, a receptor-ligand pair, regulates cortical development and lamination. Proc Natl Acad Sci USA 108:12925-12930. https://doi.org/10.1073/pnas.11048 21108

24. Langenhan T, Piao X, Monk KR (2016) Adhesion G proteincoupled receptors in nervous system development and disease. Nat Rev Neurosci 17:550-561. https://doi.org/10.1038/ nrn.2016.86

25. Langenhan T, Aust G, Hamann J (2013) Sticky signaling-adhesion class $\mathrm{G}$ protein-coupled receptors take the stage. Sci Signal 6:re3. https://doi.org/10.1126/scisignal.2003825

26. Hamann J, Aust G, Araç D, Engel FB, Formstone C, Fredriksson $R$ et al (2015) International Union of Basic and Clinical Pharmacology. XCIV. Adhesion G protein-coupled receptors. Pharmacol Rev 67:338-367. https://doi.org/10.1124/pr.114.009647

27. Musa G, Engel FB, Niaudet C (2016) Heart development, angiogenesis, and blood-brain barrier function is modulated by adhesion GPCRs. Handb Exp Pharmacol 234:351-368. https://doi. org/10.1007/978-3-319-41523-9_16

28. Niaudet C, Hofmann JJ, Mäe MA, Jung B, Gaengel K, Vanlandewijck $M$ et al (2015) Gpr116 receptor regulates distinctive functions in pneumocytes and vascular endothelium. PLoS ONE 10:e0137949. https://doi.org/10.1371/journal.pone.0137949

29. Bridges JP, Ludwig M-G, Mueller M, Kinzel B, Sato A, Xu Y et al (2013) Orphan G protein-coupled receptor GPR116 regulates pulmonary surfactant pool size. Am J Respir Cell Mol Biol 49:348-357. https://doi.org/10.1165/rcmb.2012-0439OC

30. Yang MY, Hilton MB, Seaman S, Haines DC, Nagashima K, Burks CM et al (2013) Essential regulation of lung surfactant homeostasis by the orphan $\mathrm{G}$ protein-coupled receptor GPR116. Cell Rep. 3:1457-1464. https://doi.org/10.1016/j.celre p.2013.04.019

31. Fukuzawa T, Ishida J, Kato A, Ichinose T, Ariestanti DM, Takahashi $\mathrm{T}$ et al (2013) Lung surfactant levels are regulated by IgHepta/GPR116 by monitoring surfactant protein D. PLoS ONE 8:e69451. https://doi.org/10.1371/journal.pone.0069451

32. Valenzuela DM, Murphy AJ, Frendewey D, Gale NW, Economides AN, Auerbach W et al (2003) High-throughput engineering of the mouse genome coupled with high-resolution expression analysis. Nat Biotechnol 21:652-659. https://doi.org/10.1038/ nbt822 
33. Chrostek A, Wu X, Quondamatteo F, Hu R, Sanecka A, Niemann $\mathrm{C}$ et al (2006) Rac1 is crucial for hair follicle integrity but is not essential for maintenance of the epidermis. Mol Cell Biol 26:6957-6970. https://doi.org/10.1128/MCB.00075-06

34. Sörensen I, Adams RH, Gossler A (2009) DLL1-mediated Notch activation regulates endothelial identity in mouse fetal arteries. Blood 113:5680-5688. https://doi.org/10.1182/blood-2008-08174508

35. Srinivas S, Watanabe T, Lin CS, William CM, Tanabe Y, Jessell TM et al (2001) Cre reporter strains produced by targeted insertion of EYFP and ECFP into the ROSA26 locus. BMC Dev Biol $1: 4$

36. Kisanuki YY, Hammer RE, Miyazaki J, Williams SC, Richardson JA, Yanagisawa M (2001) Tie2-Cre transgenic mice: a new model for endothelial cell-lineage analysis in vivo. Dev Biol. 230:230 242. https://doi.org/10.1006/dbio.2000.0106

37. de Lange WJ, Halabi CM, Beyer AM, Sigmund CD (2008) Germ line activation of the Tie 2 and SMMHC promoters causes noncellspecific deletion of floxed alleles. Physiol Genom 35:1-4. https:// doi.org/10.1152/physiolgenomics.90284.2008

38. Lu S, Liu S, Wietelmann A, Kojonazarov B, Atzberger A, Tang C et al (2017) Developmental vascular remodeling defects and postnatal kidney failure in mice lacking Gpr116 (Adgrf5) and Eltd1 (Adgrl4). PLoS ONE 12:e0183166. https://doi.org/10.1371/journ al.pone. 0183166

39. Armulik A, Genové G, Mäe M, Nisancioglu MH, Wallgard E, Niaudet $C$ et al (2010) Pericytes regulate the blood-brain barrier. Nature 468:557-561. https://doi.org/10.1038/nature09522

40. Huang X, Shen Y, Zhang Y, Wei L, Lai Y, Wu J et al (2013) Rac1 mediates laminar shear stress-induced vascular endothelial cell migration. Cell Adhes Migr 7:462-468. https://doi.org/10.4161/ cam. 27171

41. Joseph C, Quach JM, Walkley CR, Lane SW, Lo Celso C, Purton LE (2013) Deciphering hematopoietic stem cells in their niches: a critical appraisal of genetic models, lineage tracing, and imaging strategies. Cell Stem Cell 13:520-533. https://doi.org/10.1016/j. stem.2013.10.010

42. Baeyens N, Larrivée B, Ola R, Hayward-Piatkowskyi B, Dubrac A, Huang B et al (2016) Defective fluid shear stress mechanotransduction mediates hereditary hemorrhagic telangiectasia. J Cell Biol 214:807-816. https://doi.org/10.1083/jcb.20160 3106

43. Scholz N, Gehring J, Guan C, Ljaschenko D, Fischer R, Lakshmanan V et al (2015) The adhesion GPCR latrophilin/CIRL shapes mechanosensation. Cell Rep. 11:866-874. https://doi. org/10.1016/j.celrep.2015.04.008

44. Scholz N, Guan C, Nieberler M, Grotemeyer A, Maiellaro I, Gao $S$ et al (2017) Mechano-dependent signaling by Latrophilin/CIRL quenches cAMP in proprioceptive neurons. eLife. https://doi. org/10.7554/elife.28360

45. Tang X, Jin R, Qu G, Wang X, Li Z, Yuan Z et al (2013) GPR116, an adhesion G-protein-coupled receptor, promotes breast cancer metastasis via the Goq-p63RhoGEF-Rho GTPase pathway. Cancer Res 73:6206-6218. https://doi.org/10.1158/0008-5472. CAN-13-1049

46. Nohata N, Uchida Y, Stratman AN, Adams RH, Zheng Y, Weinstein BM et al (2016) Temporal-specific roles of Rac1 during vascular development and retinal angiogenesis. Dev Biol 411:183194. https://doi.org/10.1016/j.ydbio.2016.02.005

47. Shen W, Fruttiger M, Zhu L, Chung SH, Barnett NL, Kirk JK et al (2012) Conditional Müllercell ablation causes independent neuronal and vascular pathologies in a novel transgenic model. J Neurosci Off J Soc Neurosci. 32:15715-15727. https://doi. org/10.1523/JNEUROSCI.2841-12.2012

48. Johnson V, Xiang M, Chen Z, Junge HJ (2015) Neurite mistargeting and inverse order of intraretinal vascular plexus formation precede subretinal vascularization in Vldlr Mutant Mice. PLoS ONE 10:e0132013. https://doi.org/10.1371/journal.pone.0132013

49. Gass JDM, Agarwal A, Lavina AM, Tawansy KA (2003) Focal inner retinal hemorrhages in patients with drusen: an early sign of occult choroidal neovascularization and chorioretinal anastomosis. Retina Phila Pa 23:741-751

Publisher's Note Springer Nature remains neutral with regard to jurisdictional claims in published maps and institutional affiliations. 